\title{
Die Macht der Propaganda im Ukrainekonflikt
}

\author{
Anna Sarmina
}

\section{Abstract}

Der vorliegende Beitrag gibt einen Überblick über die Geschichte, den Begriff und Hauptmerkmale von Propaganda, deren Hauptfunktion auf die Steuerung der sozialen Systeme und auf Manipulationen der Öffentlichkeit ausgerichtet ist. Am Beispiel des auf Propagandamechanismen gründenden Ukraine-Konflikts, der seit 2014 ein heftig diskutiertes Thema bleibt, wurde der Einsatz klassischer Propagandatechniken und-methoden analysiert. Aufgezeigt wird die aktuelle politische Situation in Russland, durch die sich die Politik der Einflussnahme des Kremls erklären lässt.

Das 21. Jahrhundert ist nicht nur durch eine rasche Entwicklung von Technologien und Digitalisierung gekennzeichnet, sondern auch durch die schnelle Verbreitung von Informationen aller Art, die nicht selten mit bestimmten Zielen und Absichten in die Welt gesetzt werden. Solche Ziele können sowohl positive Veränderungen mit sich bringen als auch großen Schaden anrichten. In Zeiten der Globalisierung sind die Grenzen der Informationsflut verschwommen, Information hat einen globalen Charakter. Dadurch wird sie zur wichtigen und sogar strategischen Ressource, die in der Gesellschaft produziert und konsumiert wird und soziale Veränderungen verursacht.

Diese Prozesse haben dazu beigetragen, dass ausländische Einflussnahme auf die Innenpolitik anderer Staaten nicht nur möglich, sondern auch konventionell wurde. Eine führende Rolle in dieser Hinsicht nimmt Russland ein, das in zahlreichen Ländern Einfluss genommen hat, darunter die USA, Deutschland, Frankreich und Großbritannien. Auch die Ukraine erlebt seit 2014 eine fortschrittliche Form hybrider Kriegsführung nach atypischen Mustern, die in diesem Krieg aus bewaffneten Konflikten, Gewalt und Propaganda als unsichtbare Macht besteht. Die ganze Welt wird mit der schärfsten Propagandaoffensive konfrontiert, auch im Ukraine-Konflikt spielt Propaganda mit ihren Techniken und Mechanismen eine wichtige Rolle, weil sie rechtswidrige Handlungen tarnen und Tatsachen vertuschen kann. Dabei spielen nichtmilitärische Mittel eine viel wichtigere Rolle als Waffen. 
Eigentlich sind Informationskampagnen, die auf Propagandamechanismen gründen, keineswegs ein neues Phänomen, denn Information war schon immer ein Teil von Konflikten und diente als Waffe. Dies veranschaulichen historische Beispiele wie das trojanische Pferd oder die Zimmermann-Depesche gut. Im 21. Jahrhundert, in der Ära der Digitalisierung, erlebt der Informationskampf aber seine Blütezeit. Er zeichnet sich durch den Einsatz von Kommunikationstechnologien sowie durch Cyber-Attacken und die zunehmende Bedeutung der Zivilgesellschaft aus. In verschiedenen Ländern nehmen diese unterschiedliche Formen an, außerdem werden verschiedene Techniken verwendet. Digitalisierungsprozesse und die Verbreitung des Internets haben der Entwicklung von Propaganda einen neuen Anstoß und somit neue Möglichkeiten für die Kontrolle der Massen gegeben.

Seit jeher steht die Menschheit unter dem Einfluss von verschiedenen Systemen der sozialen Steuerung. Solche Systeme gründen vor allem auf Regeln und Normen, gleichzeitig können solche Regeln sowohl offen und demokratisch als auch versteckt und brutal sein. Seit jeher existieren effektive Steuerungsmethoden, die auf den ersten Blick nicht so brutal sind wie Foltermethoden zu Inquisitionszeiten und im Vergleich zu Drangsalierung und Torturen durch einen milden Einfluss auf das Bewusstsein das gleiche Ziel erreichen können. Propaganda ist ein klassisches Beispiel dafür, dicht gefolgt von Religion und Ideologie. Sie können miteinander kombiniert oder autonom eingesetzt werden, und jede einzelne Methode kann über Jahrzehnte, sogar Jahrhunderte wirksam bleiben.

Die Gesellschaft aber entwickelt sich ununterbrochen. Die Systeme der sozialen Steuerung erleben parallel dazu eine Evolution, und so sind neue Methoden und neue Herangehensweisen zur Steuerung der Menschen erforderlich, die an die neue Gesellschaft angepasst sind und in dieser Gesellschaft erfolgreich und relativ natürlich funktionieren können. Da die moderne Periode der Entwicklung unserer Öffentlichkeit durch die entscheidende Rolle der Information geprägt ist sowie durch die rasche Entwicklung des Internets und der sozialen Medien, bedingt das die Notwendigkeit, qualitativ neue Herangehensweisen an die Steuerung der Gesellschaft auszuarbeiten. Dabei müssen Prozesse berücksichtigt werden, die großen Einfluss auf die Etablierung neuer Regeln, Normen sowie Verhaltensweisen ausüben. Die besten neuen Dinge sind vergessene alte. Gerade deswegen dominieren klassische Steuerungsmethoden, auch wenn sie neue, modernere Formen haben.

In der Öffentlichkeit wird der Begriff Propaganda mit totalitären Regimen assoziiert und folglich negativ wahrgenommen. Ungeachtet dessen werden im Allgemeinen unter Propaganda intensive kommunikative 
Prozesse verstanden, die darauf abzielen, das Verhalten des Publikums zu verändern. Kommunikationstheoretisch bildet Propaganda eine besondere Form der systematisch geplanten Massenkommunikation, die nicht informieren oder argumentieren, sondern überreden oder überzeugen möchte (Bussemer, 2008).

Seiner Herkunft nach hat dieser Begriff eine völlig neutrale Bedeutung, die dem kirchlichen Bereich entstammt. Er hat sich aus dem Congregatio de propaganda fide, dem Namen einer 1622 in Rom gegründeten »päpstlichen Gesellschaft zur Verbreitung des Glaubens« entwickelt (Bussemer, 2008). Im 17. Jahrhundert war Propaganda ein Instrument der christlichen Missionsarbeit und der Gegenreformation. Doch schon im 18. Jahrhundert wurde Propaganda als Kommunikationstechnik gesehen und entsprechend negativ konnotiert. Zur Zeit der Französischen Revolution wurde sie als Technik der Verbreitung von Ideologien und als wichtiges Mittel der Agitation eingesetzt. Erst im 20. Jahrhundert wurde von der neuen Propaganda gesprochen, die zum unverzichtbaren Bestandteil der Kriegsführung geworden war (Bussemer, 2008).

Im 21. Jahrhundert wird schon von einem engeren und weiteren Propagandabegriff gesprochen. Der engere Begriff bestimmt die Diskussionen um Psychological Warfare, Public Diplomacy und Information Operations in der globalen Informationsumwelt. Der weitere Begriff bezeichnet die professionell geplante Meinungswerbung in Demokratien (Bussemer, 2008). Ein enger und ein weiter Propagandabegriff koexistieren also: Während der enge Begriff mit Propaganda mit totalitären Strukturen der Informationskontrolle und der Unterdrückung der öffentlichen Meinung verknüpft und der Propaganda so erhebliche Wirkungsmacht zuschreibt, geht der weite Propagandabegriff von einer beinahe ubiquitären Präsenz von Propaganda in allen Gesellschaftssystemen aus, unterstellt aber im Gegenzug schwächere Wirkungen (Bussemer, 2008).

Eine deutlich negative Konnotation hat der Begriff Propaganda erst infolge der Herrschaft der politischen Regime von Hitler sowie Stalin bekommen. Durch die totalitäre Politik in der UdSSR und in Deutschland wurde er kompromittiert. Im Abendland wird das Wort Propaganda mit solchen Institutionen wie dem Reichsministerium für Volksaufklärung und Propaganda in Deutschland, ergo mit der Kommunikationspolitik der Nationalsozialisten oder mit einzelnen Personen wie Joseph Goebbels assoziiert, in postsowjetischen Ländern eher mit dem kommunistischen Regime. Auf jeden Fall ist Propaganda für viele ein Teil der Geschichte, der in Vergessenheit geraten ist. 
Doch auch heute bleibt Propaganda ein effektives Steuerungsmittel, welches unter dem Begriff Propaganda 2.0 vielfach eingesetzt wird (Pocheptsov, 2017). Das Phänomen betrifft zahlreiche Lebensbereiche und nimmt einen wichtigen Platz in der Kommunikationswissenschaft ein. Werbung und Public Relations unterscheiden sich jedoch dadurch, dass sie die Existenz von Alternativen erlauben, während Propaganda gegen Alternativen kämpft.

Die Meinung der Massen ist offensichtlich formbar, sodass ihre neu gewonnene Kraft in die gewünschte Richtung gelenkt werden kann. Unsere heutige Gesellschaftsstruktur würde ohne diese Praxis nicht funktionieren. Wann immer etwas von allgemeiner Bedeutung unternommen werden soll, sei es in den Bereichen Politik, Finanzen, Industrie, Landwirtschaft, Wohltätigkeit, Bildung oder auf anderen gebieten, dient Propaganda den unsichtbaren Herrschern als Mittel der Durchsetzung. (Bernays, 2007, 18)

Propaganda ist eine Form der Kommunikation, die die Formierung der Überzeugungen und Einstellungen sowie des Glaubens der Bevölkerung in Bezug auf die Lösung bestimmter Probleme beeinflusst. Propaganda ist also gerichtet auf die positive oder negative Wahrnehmung bestimmter Religionen, Ideologien, politischer Kräfte und all das auf dem Niveau des Massenbewusstseins. Sie wirkt auf emotionalem und nicht auf rationalem Niveau.

Trotz der demokratischen Regierungsform bleibt die Rolle der Propaganda bedeutend, weil sie ein effektives Mittel für Manipulationen der Öffentlichkeit ist. »Die bewusste und zielgerichtete Manipulation der Verhaltensweisen und Einstellungen der Massen ist ein wesentlicher Bestandteil demokratischer Gesellschaften « (Bernays, 2007, 11). Propaganda kann somit natürlich in einer demokratischen Gesellschaft funktionieren und ihre Hauptaufgaben produktiv erfüllen.

Jedoch ist auch die verdeckte, gefährliche Propaganda noch existent, sie verbirgt sich im Dickicht des Internets und ist heutzutage nicht weniger verbreitet als zu Zeiten des Dritten Reichs. Die sozialen Medien haben einen Raum geschaffen, in dem illiberale Werte einschließlich Rassismus, Frauenfeindlichkeit und Homophobie ungezügelt propagiert werden (Pocheptsov, 2019a). Sie sind zum neuen aggressiven Werkzeug der Politik geworden, zum Beispiel durch den Einsatz automatischer Twitter-Accounts, sogenannter Social Bots, die den virtuellen Raum mit Propaganda fluten. Eine weitere Gefahr sind »Fake News«, deren Verbreitung durch die sozialen Netzwerke die demokratische Meinungsbildung beeinträchtigt.

Ungeachtet dessen, dass wir heutzutage nicht mehr in einem totalitären System leben, sondern in der einen oder anderen Form der Demokratie, schließt das nicht aus, dass auch Demokratien neue Methoden von Propaganda verlangen, die frühere harte Methoden der Regelung ersetzen. 
Egal ob früher oder heute, im Großen und Ganzen wird unter Propaganda ein bewusster Versuch verstanden, Menschen zu überzeugen, dass sie wie erwünscht denken und sich verhalten. Die Ära der Digitalisierung hat diese Aufgabe viel leichter gemacht. Momentan findet der Prozess der Transformation von Menschen statt, weil ein sogenannter homo digitalis seine mentalen Funktionen auf technische Mittel überträgt (Montag, 2018). Einerseits wird die menschliche Existenz dadurch erleichtert: Man kann Antworten auf nahezu alle Fragen problemlos online bekommen und die fertigen Antworten werden schnell und ohne weitere Überlegungen rezipiert. Das führt dazu, dass man auf fertige Antworten wartet, damit rechnet, dass jemand anderes für einen selbst denkt, und schließlich Informationen inklusive fertiger Schlussfolgerung bekommt. Andererseits versteckt sich hier eine Gefahr, dass man kognitiv angreifbar wird.

Diese Faktoren bedingen die Notwendigkeit, Propaganda als soziales Phänomen zu erforschen und Besonderheiten der Propaganda ans Licht zu bringen. In letzter Zeit ist das wachsende Interesse an Propaganda zu bemerken, weil sich ihre Rolle in der modernen, digitalen Welt deutlich verändert hat. Dieser Trend stützt sich auf verschiedene Faktoren, darunter die globale Rolle der Information, den sozialen Wandel und die wachsende Bedeutung der sozialen Netzwerke, die zu einer der wichtigsten Nachrichtenquellen für besonders empfängliche und sensible jüngere Zielgruppen geworden sind (Reuters Institute Digital News Report, 2019). Ausschlaggebend für diese Studie ist aber die Tatsache, dass die Flut der propagandistischen Produkte zum Katalysator von Kriegen geworden ist und damit ein mächtiges Instrument der Kriegsführung und des Ausübens von Terror ist. Vor dem Ukraine-Konflikt wurden im Informationsraum der Ukraine ideologische Klischees verbreitet, die die Öffentlichkeit von innen zersplitterten und Unzufriedenheit erzeugten (Hofmeisterova et al., 2018).

Wichtig ist dabei die Tatsache, dass der Einsatz von Propaganda eine langjährige erfolgreiche Geschichte in vielen Ländern hat. Propaganda verfügt seit jeher über ein wirksames und ganz erfolgreiches Instrumentarium. Auch in ihrer modernen Variante, die wir als Propaganda 2.0 bezeichnen, bleibt sie ein wichtiges Instrument für den Einfluss auf die Öffentlichkeit und das Ausmaß ihrer Wirkung ist oft größer als erwartet (Pocheptsov, 2017). In der Unauffälligkeit liegt der Schlüssel zum Erfolg, denn je unsichtbarer ein solches Instrument ist, desto mächtiger wirkt es.

Richard Nixon hielt es für sinnvoller, einen Dollar in Propaganda zu investieren, als zehn Dollar in die Modernisierung der Waffen und Ausrüstung, weil Propaganda im Unterschied zu Waffen jeden Tag und jede Stunde wirke (Kopacek, 2004). 
Propaganda in der UdSSR war äußerst erfolgreich und wirksam, sie übte einen psychologischen Einfluss auf die Moral der Bevölkerung aus (Pocheptsov, 2018). Da die West-Alliierten und die Sowjetunion nach dem Krieg verschiedene Wege beschritten, entwickelten sich auch unterschiedliche Formen von Propaganda. Werbung und PR, die sich rasch in Europa verbreiteten, hatten zum Ziel, das Konsumverhalten zu beeinflussen und potenzielle Konsumenten zum Kauf zu animieren. So wurde mithilfe von kommunikativen Methoden ein Zuwachs der Warenproduktion möglich. Kommerzielle Propaganda und PR waren für die Sowjetunion belanglos, weil das Land unter ständigem Warenmangel litt. In den Vordergrund des Lebens in der UdSSR wurde die politische Propaganda gestellt. Sowjetische Propaganda ist durch eine ganze Reihe Merkmale gekennzeichnet (Pocheptsov, 2018, 2018a). Sie hatte eine laute Stimme und gleichzeitig einen stillen Einfluss. Die laute Stimme wurde an die Massen gerichtet und der stille Einfluss an ein Individuum. Das bestätigen zwei Hauptmerkmale der damaligen totalitären Propaganda: mehrmalige Wiederholung sowie Repressalien beziehungsweise Vergeltungsmaßnahmen. Als andere Merkmale der sowjetischen Propaganda sind folgende zu nennen: Die Propaganda war monologisch, sie wurde durch die ganze Infrastruktur des Staates unterstützt (Bildung, Literatur, Kunst und sogar Ordnungsbehörden usw.), sie gehörte als Pflichtteil zum Leben dazu und wurde mit verschiedenen Mitteln eingeführt und in den Nachrichten verbreitet. Alternative Informationen waren nicht zugänglich. Die Propaganda beruhte auf Helden. Die Gegenüberstellung von Freund und Feind war notwendig. Die damalige Weltsicht, in der gute und schlechte Persönlichkeiten, gute und schlechte Taten sowie gute und schlechte Ideen deutlich voneinander abgegrenzt wurden, entstand schließlich mithilfe der Propaganda (Pocheptsov, 2018, 2018a). Darüber hinaus ist die sowjetische Propaganda von binären Unterscheidungen wie »wir und sie«, »gut und böse«, »gerecht und ungerecht, »unschuldig und schuldig «, »sinnvoll und sinnlos «, »gesittet und ungesittet « gekennzeichnet, die eine binäre Weltanschauung prägen (Pocheptsov, 2018; 2018a).

Ein weiteres nicht weniger wichtiges Merkmal der Propaganda ist, dass sie sowohl mit der Sprache als auch mit den Bildern arbeitet, weil die visuelle Ästhetik mehr emotionale Wirkung hat. Harold D. Lasswell definiert Propaganda als »management of collective attitudes by the manipulation of the significant symbols « (Lasswell, 1927, 627). Unter Symbolen werden Bilder und andere non-verbale Mittel verstanden, was auf semiotische Aspekte der Propaganda hinweist. Propaganda arbeitet über und mit Bildern, die manipuliert werden, sodass im Rezeptionsprozess neue Verknüpfungen 
zwischen vorhandenen positiven oder negativen Einstellungen und bestimmten Sachverhalten hergestellt werden. Dieser Prozess funktioniert entweder durch die Manipulation semiotischer Kopplungen oder durch die Verfälschung von Fakten (Bussemer, 2008). Für die besondere Bedeutung der visuellen Objekte spricht auch der erfolgreiche Einsatz von Instagram (Wong, 2018) als bestes Online-Instrument der russischen Propaganda.

Obwohl Propaganda mit der Zeit einen Entwicklungsprozess erlebt und neue Merkmale bekommen hat, bleibt das Ziel der Propaganda gleich, andere Menschen davon zu überzeugen, zu einer spezifischen Frage eine spezifische Haltung einzunehmen und auch nach dieser Überzeugung zu handeln (Bernays, 2007).

Die neue Propaganda oder Propaganda 2.0 entwickelte sich aus den Theorien von Bernays (2007) und Ellul (1927). Bernays betont, dass sich die neue Propaganda nicht mehr mit dem Individuum oder der Gesellschaft als Ganzes befasst, sie sieht den Einzelnen als sensiblen Punkt des Organismus »Gesellschaft « (Bernays, 2007). Die Hauptbesonderheit der modernen Propaganda sieht Georgii Pocheptsov darin, dass sie durch ein hohes Niveau an Kreativität gekennzeichnet ist. Propaganda 2.0 ist Quasi-Kunst, die das Weltbild schafft, das für uns attraktiv scheint. Sie wird in Kultur, Literatur und Kunst realisiert und Zuschauer können die propagandistischen Methoden nicht mehr erkennen. Diese Art von Propaganda wird professionell vorbereitet, für sie ist Ästhetik sehr wichtig. Massenmedien greifen in der letzten Zeit in dieses Spiel ein: Sie tarnen propagandistische Mitteilungen in Nachrichten, Dokumentarfilmen und Reality-Shows, um den Widerstand des Publikums herabzusetzen und notwendige Informationen in Massen zu verbreiten (Pocheptsov, 2018c). Da Propaganda sowohl zum Krieg als auch zum Frieden berufen kann, soll noch mehr Kreativität dabei helfen, wenn der Krieg propagiert wird. Die Wortwahl spielt hier zum Beispiel eine große Rolle.

Russische Propaganda in der Ukraine ist von Merkmalen der klassischen sowjetischen Propaganda gekennzeichnet, vor allem spielt sie mit der Gestalt des Feindes (Pocheptsov, 2018). Der bewaffnete Konflikt zwischen der Ukraine und Russland ist ein Teil des propagandistischen Krieges, dessen Held Russland ist, weil es als Retter des ukrainischen Volkes dargestellt wird. Als deutliche Feinde treten in der Ukraine agierende Nazis, sogenannte Banderivtsi (Anhänger des Nationalhelden und Freiheitskämpfers Stepan Bandera) auf. Auch alte Schablonen, die aus den Zeiten des Kalten Krieges stammen, werden wieder ins Leben gerufen, weil die Kriege, die heutzutage geführt werden, auch auf dieser Ideologie gründen. Die klassischen propagandistischen Methoden, die Georgii Pochepstov als 
Metapropaganda bezeichnet, sind nicht tot, sie wachsen und gedeihen. Unter dem Begriff Metapropaganda versteht er die Verbindung von Ideologie und Propaganda, wobei Propaganda viel stärker ist als Ideologie (Pocheptsov, 2018b). Den Unterschied zwischen Propaganda und Metapropaganda sieht er darin, dass ungeachtet der Tatsache, dass die beiden auf eine Transformation des Weltmodells abzielen, Propaganda viel offener und direkter verbreitet wird, und Metapropaganda dies eher indirekt und unterbewusst tut, sich den Informationen anpasst und einen hohen Grad an Kreativität aufweist (Pocheptsov, 2018c).

Es sei erwähnt, dass das Fernsehen in der UdSSR ein Sprachrohr von Propaganda war, weil die Informationen, die im Fernsehen übertragen wurden, als unangefochtene Wahrheit galten. Diese Vorstellung ist bei Menschen älterer Generationen bis heute fest in ihrem Bewusstsein verankert vor allem deswegen, weil sie sich an den Gedanken gewöhnt haben, dass Vertrauen in staatliche Medien unumstritten ist. Heutzutage spielt Fernsehen eine wichtige Rolle als Informationsquelle für eine große Zahl der russischen Bürger (Levada-Center, 2019), weswegen immer mehr neue Medienprodukte angeboten werden, die eine Art Infotainment sind und Einfluss üben wollen (Postman, 1988). Der letzten Befragung nach (Stand Mai 2019) verbringen 79 Prozent der russischen Bürger ihre Zeit mindestens einmal pro Woche vor dem Fernseher. Diese Aktivität steht an der Spitze der Umfrage (Levada-Center, 2019). Die Kultur des Fernsehens in Russland wird sorgfältig gepflegt. Immer mehr propagandistische Produkte verschiedener Gattungen werden dem Publikum angeboten, um das gewünschte notwendige Modell der Wirklichkeit im Bewusstsein der Bürger zu schaffen. Die Macht des Landes wird zum Beispiel dadurch betont, dass mithilfe der Dokumentarserie The Putin Interviews das Bild eines mächtigen und starken Staatsoberhauptes geschaffen wird. Visuelle Objekte erhöhen den Grad der Emotionalität, gleichzeitig dominiert die emotio über die ratio, worauf sich jede Art von Propaganda gründet (Bussemer, 2008).

Nach dem gleichen Verfahren wird auch das Bild der Ukraine für die russischen Bürger kreiert. Tagtäglich werden Nachrichten und Informationen verschieden modifiziert und sowohl im Fernsehen als auch auf OnlineNachrichtenportalen präsentiert. »Die Ukraine ist seit fünf Jahren der Hauptdiskurs in Russland [...]. Sich aus den russischen Armen entrissen, bleibt sie ein scheinbarer Schmerz Russlands«, sagt die russische Politologin Lilia Shevtsova in ihrem Artikel mit dem Titel »Ukraine als russische Obsession« (Shevtsova, 2019). Diese Metapher spiegelt reale Verhältnisse zwischen den beiden Staaten wider, wobei sich Russland als verschmähte Geliebte verhält, die neue Rachepläne ausheckt. In Wirklichkeit hatte die 
Ukraine die Absicht, sich aus dem blinden Gehorsam und Konformismus des homo sovieticus zu befreien (Justenhoven, 2018), was seitens Russlands als ein Akt des Ungehorsams gesehen wurde.

Wenn man in russischen Informationsportalen nach Schlüsselthemen sucht, haben viele Erwähnungen über die Ukraine negative Konnotationen. $\mathrm{Zu}$ solchen Schlüsselthemen, die russische Propaganda über die Ukraine widerspiegeln, gehören folgende (Stand Mai 2018): In der Ukraine herrscht ein Bürgerkrieg (33 Prozent des Contents); die Ukraine ist ein gescheiterter Staat (22 Prozent); Russland leistet Hilfe für Donbass (15 Prozent); in der Ukraine herrscht Russophobie (10 Prozent); in der Ukraine sind Faschisten und Radikale an der Macht (7 Prozent); die Ukraine ist eine Marionette des Westens, der jahrzehntelang ergebnislos Russland erobern wollte (6 Prozent) (Pocheptsov, 2018d). Außerdem werden Ukrainer selbst als Untermenschen bezeichnet. Russland macht einen Feind aus der Ukraine, um eigene illegale Taten zu rechtfertigen, sich selbst zu rechtfertigen und so gleichzeitig ein Scheinbild zu schaffen, dass der Ukraine-Konflikt ausschließlich zwischen den Ukrainern verläuft (Pocheptsov, 2018e). Als ein gutes Beispiel kann hier die Situation im Juni 2019 im Parlament Georgiens dienen. Nach Ausschreitungen vor dem Parlament in Tiflis infolge des Auftritts eines russischen Abgeordneten hat Russland wieder die USA und die Ukraine beschuldigt, dass sie dieses Chaos Engineering geschaffen hätten (Shimaev, 2019), was sich nicht zuletzt dadurch erklären lässt, dass sechs post-sowjetische Länder, darunter auch Georgien und die Ukraine, schon seit zehn Jahren gegen Russland im Rahmen der Östlichen Partnerschaft ihre Freundschaft pflegen (Vostochnoe partnerstvo, 2019).

Fernsehsendungen aller Gattungen geben bis heute Antworten auf alle Fragen, sodass die Menschen bequem und glücklich leben könnten und sich keine unnötigen Gedanken machen müssten (Pocheptsov, 2018c). Diese Scheinwelt besteht ausschließlich aus Lösungen, es gibt keine Schwierigkeiten. Jedoch leben die Menschen in dieser Scheinwelt nicht ihr eigenes Leben, sondern das Leben der Anderen. Fernsehen ist ein Instrument, das Propaganda, Ideologie und Amüsement zu einer untrennbaren Einheit verbindet, die die reale Welt durch eine propagandistische ersetzt (Pocheptsov, 2018b). Interpretationen der Tatsachen auf dem Bildschirm haben das Potenzial, die Realität im Großen und Ganzen zu ersetzen. Fernsehprodukte, nämlich Dokumentarfilme, Talk-Shows und Serien formen die Denkweise des Volkes. Fernsehen spielt mit Emotionen der Menschen und visualisiert notwendige Informationen, beispielsweise wie abschreckend das Leben im Westen und in Amerika angeblich ist, oder präsentiert immer neue Horrorgeschichten aus der Ukraine. Einige Erklärungen, die aus dem Fernseher 
ertönen, sind primitiv, doch sie wirken sehr erfolgreich, wie zum Beispiel eine klassische russische Formel: »Die Ukraine ist schlecht, weil da der Westen handelt« (Pocheptsov, 2018d). Diese negative Implikation gibt den Anstoß für negative Schlussfolgerungen (Pocheptsov, 2018d). Die Mehrheit der Menschen braucht leichte Erklärungen für die schwierigen Fragen. Dies ist gerade der Fall, wenn von der Ukraine gesprochen wird. Früher wurde behauptet, dass Russen und Ukrainer Brüder sind. Jetzt sind die Ukrainer zu Feinden geworden. Von heute auf morgen wären solche Veränderungen unbegreiflich, deswegen: Je leichter die Erklärung ist, desto schneller wird sie wahrgenommen. Eine einfache Erklärung in diesem Sinne ist die folgende: »In der Ukraine leben Russischsprachige, die den Schutz brauchen." Georgii Pocheptsov (2018e) spricht in diesem Zusammenhang von »kognitiven Angriffen«, die die kognitive Transformation des Massenbewusstseins zum Ziel haben.

Solche Inhalte in russischen Medien stellen eine ganze Reihe der Beispiele des Einsatzes des klassischen Propagandamodells dar (Herman \& Chomsky, 2002), wobei die Informationen sorgfältig gefiltert werden. Das Verfahren, die Aufmerksamkeit auf die Probleme des Nachbarlandes zu lenken, um der Lösung eigener Probleme zu entgehen und die Aufmerksamkeit der Öffentlichkeit davon abzulenken, ist auch eine klassische Methode der russischen Propagandamaschine. Andererseits ist das wieder ein Spiel mit binären Gegenüberstellungen: Das Leben in der Ukraine sei sehr schlecht, in Russland im Gegensatz dazu sehr gut. Dabei sieht das Volk den Balken im eigenen Auge nicht, aber den Splitter in dem des Fremden, wie es schon in der Bibel steht, was zu mehr Handlungsfreiheit der Machthaber führt.

Um ein globales Ziel zu erreichen, ist es notwendig, alternative Informationsquellen zu sperren, damit Menschen keinen Zugang zu fremden und vermeintlich falschen Ideen haben. Zur Zeit der Sowjetunion spielte der Eiserne Vorhang diese Rolle, heutzutage ist es die absichtliche Begrenzung und sogar Isolation des Internets. Es geht eigentlich nicht um ein nationales Filtersystem, sondern um Zensur, die das Funktionieren von Propaganda innerhalb des Landes garantieren soll. Die Nutzung von verbotenen Quellen wird dokumentiert, sodass sich die Menschen wieder beobachtet und verfolgt fühlen sollen. Dieser Schritt ist auch der Rücktritt zu alten und klassischen Methoden der Steuerung, die zu sowjetischen Zeiten ein unentbehrlicher Teil des Totalitarismus waren. Damals gab es keinen Platz für Unstimmigkeiten, die Zweifel hätten entstehen lassen können. Die Trennung vom globalen Netz bedeutet die Unmöglichkeit der Existenz eines alternativen Weltbildes und gleichzeitig das Aufzwingen eigener Werte 
(Pocheptsov, 2019). Da die Importanz des Internets ununterbrochen steigt, verbringen die Menschen einen bedeutenden Teil ihres Lebens online. Sehr vielsagend ist die Tatsache, dass Russland im Ranking des World Press Freedom Index den 149. Platz von 180 Ländern belegt hat. Der Kreml verstärkt den Druck auf die Medien und auf das Internet (Reporters Without Borders 2019a; 2019b) und schafft die Illusion der Pressefreiheit. Das Gespenst des Maidans wandere durch Russland, sagen die Experten, und es bestehe die Gefahr, dass Russen auch diesen Weg bevorzugen werden (Pavlenko, 2019). Deswegen sollen notwendige Maßnahmen rechtzeitig ergriffen werden. Das Vorgehen der Polizei gegen Demonstranten im Juli 2019 in Moskau, bei dem mehr als 1000 Personen festgenommen und schwer verletzt wurden, hat deutlich gezeigt, wie die typischen Vergeltungsmaßnahmen gegenüber Andersgesinnten aussehen, die nach der gleichen Lösung wie die Ukrainer suchen.

Darüber hinaus werden Propagandatechniken hinsichtlich der Ukraine immer intensiver eingesetzt. Obwohl Bernays (2007) behauptet, dass sich die Propagandatechniken von heute erheblich von denen unterscheiden, die vor zwanzig Jahren eingesetzt wurden, zeigen sich Änderungen im Bereich der Ökonomie. »Die Wirtschaft hat die alten Methoden im harten Wettbewerb immer mehr verfeinert, während die Politik noch an den alten Formeln festhält« (Bernays, 2007, 63).

Im Ukraine-Konflikt sind zahlreiche Beispiele des Einsatzes klassischer Propagandatechniken zu nennen. Eine breite Palette an Bezeichnungen für Ukrainer wird seitens Russlands verwendet, darunter sind folgende Beispiele: Faschisten, Neonazis, Junta, Banderivtsi. Solche negativ konnotierten Bezeichnungen oder Labels zielen darauf ab, negative Schlussfolgerungen hinsichtlich einer Idee, eines Ereignisses oder einer Person zu provozieren. Anders gesagt: Personen werden dämonisiert, das Land selbst sowie Institutionen werden in negativer Weise etikettiert. Dieses Verfahren gehört zu altherkömmlichen Propagandatechniken und wird von Lasswell als Name Calling bezeichnet. Nicht weniger auffällig ist hier der Einsatz der klassischen Propagandatechnik Transfer, die der vorher genannten Technik sehr ähnlich ist und die Diskreditierung der Ukraine zum Ziel hat.

Das Spiel mit Werten, die Verwendung von emotional gefärbten Redewendungen (vor allem Metaphern), die positive Assoziationen hervorrufen, ist ein weiterer Trick der russischen Propaganda, der auch als Glittering Generalities bezeichnet wird. Angefangen von der Krim-Annexion werden Assoziationen mit Illegalität umgangen, indem negative Bezeichnungen dank der Verwendung von positiv konnotierten Komponenten neutralisiert werden. $\mathrm{Zu}$ dieser Technik gehört auch der Ersatz von expliziten 
Bezeichnungen des Illegalen durch positive Konzepte, wie zum Beispiel die Wiedervereinigung der Krim statt des Begriffs der Annexion oder Notwehr statt Besetzung. Solche Mottos wie »wir kämpfen für die Freiheit russischsprechender Bürger«, »das gehört dem Volke« und »wir sind das Volk« sind darauf gerichtet, illegale Aktionen zu legitimieren. Ein solches Konzept wie Russkij Mir (Russische Welt, Russischer Frieden, Russische Gemeinschaft) kann inhaltlich diffus und eklektisch wirken, aber ungeachtet dessen »appelliert es an das möglichst breite Publikum [...] und bietet jedem, der auf irgendeine Weise mit der russischen Kultur oder dem russischen Staat verbunden ist, eine breite Palette identitätsstiftender Symbole an« (Justenhoven, 2018). Die Bezeichnung Novarossia (Neues Russland) deutet ziemlich direkt auf die Ausdehnungspolitik des Landes hin.

Sehr verbreitet bleiben noch bis heute Propagandatechniken, die auf Aussagen der Augenzeugen gründen. Dazu gehören beispielsweise Plain Folks, wobei es tatsächlich um die Tätigkeit von politischen Akteuren geht, die auf Schritt und Tritt die Gastrolle und Vertreter des Volkes spielen, deren Aussagen besonders glaubwürdig sein sollen; so war es ständig in den Nachrichten zu sehen, denn die Mehrheit der Bürger würde sich gerne mit dem sogenannten Otto Normalverbraucher identifizieren. Nicht einmal solche Bühnenkünstler wurden erwischt, geschweige denn ihre Ammenmärchen entlarvt. Die falschen Experten, die falschen Vertreter anderer Länder, geben ihre Einschätzungen und Prognosen ab, während sie an politischen Talk-Shows in Russland teilnehmen. Dass politische Shows im russischen Fernsehen eine manipulative Aufgabe erfüllen sollen, kann dadurch bestätigt werden, dass sie einen hohen Grad an Emotionalität zeigen. Menschen schlüpfen in Rollen von Augenzeugen, Experten oder Politikern, sie diskutieren, attackieren, sind laut und affektiv, wiederholen das Gleiche mehrmals, prügeln aufeinander ein, wodurch die Zuschauer von einer rationalen in eine emotionale Wahrnehmung wechseln (Pocheptsov, 2018e).

Eine bedeutende Rolle unter anderen Propagandatechniken spielen Testimonials - Referenzen und Aussagen berühmter Personen, die die Glaubwürdigkeit bestimmter Informationen erhöhen sollen. Diese Prominenten kommen aus verschiedenen Bereichen und verdienen ihr Geld unter anderem als Schauspieler, Ärzte und Politiker. Um das beste Ergebnis zu erzielen, soll für jede Zielgruppe ein passender, richtiger Zeuge gewählt werden, der dieser Zielgruppe gut bekannt ist und dessen Aussagen glaubwürdig sind.

Manipulationen mit Informationen stehen im Vordergrund der russischen Propaganda, weit und breit wird die Propagandatechnik Card Stacking eingesetzt, bei der nur positiven Informationen Wichtigkeit 
beigemessen wird, die negativen Informationen werden dagegen vertuscht oder verschwiegen. Rupert Lay (1977) unterscheidet vier Arten der Manipulationen mit Informationen:

- Übermitteln der unwahren Information (mit dem Ziel bewusster Täuschung);

- Übermitteln der unvollständigen Information (mit dem Ziel des Erschwerens der richtigen/wahren Nachricht);

- Übermitteln übermäßig vieler Information (mit dem Ziel des Verbergens negativer Aspekte);

- Übermitteln ungenauer Information (mit dem Ziel der Ablenkung der eventuellen Problematik).

Global gesehen dient jede Art der Verdrehung von Informationen zu Expansionszwecken der sogenannten russischen Welt (Russkij Mir). Heutzutage werden diese Manipulationstypen mit dem Oberbegriff Faking bezeichnet. Die Gutenberg-Ära hat den Umfang der Information erhöht und einen Raum für Wahrheit geschaffen. Die Ära des Internets hat unter anderem einen Raum der Lüge kreiert, der mit Fälschungen überfüllt ist. Die moderne Periode wird als Post-Truth-Ära bezeichnet, worunter »relating to or denoting circumstances in which objective facts are less influential in shaping public opinion that appeals to emotion and personal belief « (Oxford English Dictionary, 2019) verstanden werden. Es bedeutet, dass die bereitgestellten Informationen nicht genau, verdreht, voreingenommen oder fabriziert sind. Diese Post-Truth-Ära erzeugt eine ganze Reihe neuer Phänomene, wie zum Beispiel »Fake News« oder Desinformation, unter denen verzerrte Signale, die nicht mit der Wahrheit korrelieren, verstanden werden (Alcott \& Gentzkow, 2017). Neben dem sogenannten Trolling gehören »Fake News« zu erfolgreichen Propagandamechanismen dazu.

Eine tiefergehende Herangehensweise an dieses Problem macht deutlich, dass »Fake News« nicht nur falsche Informationen sind. Sie sind ein Baustoff, mit dem Ereignisse und gleichzeitig eine Realität für die Menschen geschaffen werden. »Fake News« können somit unser Kollektivgedächtnis beeinflussen, indem sie sich im Informationsraum bewegen und Mitautoren erhalten (Pocheptsov, 2018f).

Die Existenz sozialer Netzwerke hat dazu geführt, dass jeder Nutzer eigene kleine Wahrheiten schreibt, genauer gesagt das, was jemand für eine Wahrheit hält. Je häufiger diese kleinen Wahrheiten wiederholt werden, desto plausibler werden sie (Pocheptsov, 2018f). Außerdem ist die Information, die von Online-Freunden verbreitet wird, glaubwürdiger als die 
Information, die offiziell vom Staat kommt. Gerne teilen Menschen Informationen, die ihnen gefallen, ohne sie zu prüfen und auf diese Art und Weise schaffen sie Realität. Deswegen werden seitens Russland vermutlich Social Bots eingesetzt und wahrscheinlich ganze Troll-Fabriken gegründet, die propagandistische Mitteilungen im Netz verbreiten.

In der Ukraine gibt es mindestens vier aktive Webseiten, die Nachrichten evaluieren. Am bekanntesten ist StopFake.org, das von Alumni und Freiwilligen der Mohyla Journalism School in Kiew gegründet wurde. Diese Webseite wurde 2014 ins Leben gerufen, um die russische Propaganda in der Ukraine zu entlarven. Die Ukraine lebt immer noch unter Angriffen gefälschter Nachrichten, die in größerem Maße zur Manipulation der öffentlichen Meinung beitragen. Die Tätigkeit von StopFake.org zielt darauf ab, Fakten zu prüfen, Informationen zu verifizieren, falsche Nachrichten über die Ukraine zu widerlegen, um letztlich das Vertrauen der Öffentlichkeit in die Medien wiederherzustellen.

Die Ukraine ist schon seit langem zum Opfer der Einflussnahme des Kremls auf ihre Innenpolitik geworden. Diese Einmischungspolitik gründet auf klassischen Propagandatechniken, die dank der Digitalisierung und der Verbreitung des Internets neue Facetten und auch neue Grenzen sowie Möglichkeiten bekommen haben. Obwohl Propaganda so alt ist wie Methusalem, bleibt sie eine der erfolgreichsten Steuerungsmethoden des Massenbewusstseins und ist ein natürlicher Bestandteil jeder demokratischen Gesellschaft. Ungeachtet der Tatsache, dass russische Propaganda eine reiche Geschichte und große Kapazität hat, sind die Methoden und Instrumente, die von Russland gegen die Ukraine eingesetzt werden, erkennbar und verständlich. Das Streben nach Herstellung eigener Macht, nach Steuerung der Politik anderer Staaten und nach Expansion bestimmen das Globalziel der Propaganda des Kreml. Die Ukraine ist momentan auf dem Weg zu einer neuen Phase ihrer Geschichte, die sich auf europäische Werte stützt. Ihre Wahl hat das Land getroffen, und sie ist nicht mehr aufzuhalten. 


\section{Literatur}

Allcott, Hunt \& Gentzkow, Matthew (2017). Social Media and Fake News in the 2016 Election. Journal of Economic Perspectives, 31(2), 211-236. DOI: 1257/jep.31.2.211.

Bernays, Edward (2007). Propaganda: Die Kunst der Public Relations. Freiburg: Orange Press Verlag.

Bussemer, Thymian (2008). Propaganda: Konzepte und Theorien. Wiesbaden: VS Verlag für Sozialwissenschaften.

Levada-Center (2019). Dosug i razvlecheniya [Leisure ans entertainment]. Abgerufen von https://www.levada.ru/2019/07/01/dosug-i-razvlecheniya/. Abgerufen am 13. Februar 2020 .

Ellul, Jacques (1973). Propaganda. The formation of Men's Attitudes. Abgerufen von https://monoskop.org/images/4/44/Ellul_Jacques_Propaganda_The_Formation_of_ Mens_Attitudes.pdf. Abgerufen am 13. Februar 2020.

Herman, S. Edward \& Chomsky, Noam (2002). Manufacturing Consent: The Political Economy of the Mass Media. New York: Pantheon.

Hofmeysterova, Pavla; Dufkova, Katerina; Syrovatka, Ionash; Smatana, Iurai; Targalski, Ershi; Toldeshi, Beatris; ... Elinkova, Barbora (2018). Harakteristika prokremlevskoj propagandy v central'noj $i$ vostochnoj Evrope i primery kak s nej spravit'sya [Charakteristics of Pro-Kemlin Propaganda and Examples how to Cope with it]. Brno: Nesenhuti.

Justenhoven, Heinz-Gerhard (Hrsg.) (2018). Kampf um die Ukraine. Ringen um Selbstbestimmung und geopolitische Interessen. Baden-Baden: Nomos Verlagsgesellschaft.

Kopacek, Peter \& Stapleton, Larry (2004). Technology and International Stability (SWIIS 2003). A Proceedings Volume from the IFAC Workshop, Waterford, Republic of Ireland, 3-5 July 2003.

Lasswell, Harold (1927). The Theory of Political Propaganda. The American Political Science Review, 21(3), 627-631. DOI: 10.2307/1945515.

Lay, Rupert (1977). Manipulation durch die Sprache. München: Langen-Müller Verlag. Montag, Christian (2018). Homo Digitalis: Smartphones, soziale Netzwerke und das Gehirn. Wiesbaden: Springer Verlag.

Oxford English Dictionary online (2019). Abgerufen von http://www.oed.com/. Abgerufen am 13. Februar 2020.

Pavlenko, Irina (2019). Khto ne skache, toi za hram. [Who does not jump, he is for the Temple]. Abgerufen von https://zn.ua/international/hto-ne-skache-toy-za-hram319404_html. Abgerufen am 13. Februar 2020

Pochepstov, Georgii (2015). Model' propagandy Zhaka Ellyulya. [Propaganda Model of Jacques Ellul]. Abgerufen von https://ms.detector.media/ethics/manipulation/ model_propagandy_zhaka_ellyulya/. Abgerufen am 13. Februar 2020. 
Pochepstov, Georgii (2017). Propaganda 2.0 ka uspeshnaya technologiya segodnyashnego dnya. [Propaganda 2.0 as today`s successful technology]. Abgerufen von https://ms.detector.media/trends/1411978127/propaganda_20_kak_uspeshnaya_ tekhnologiya_segodnyashnego_dnya/. Abgerufen am 13. Februar 2020.

Pochepstov, Georgii (2018). Propaganda sovetskaya i nesovetskaya. [Soviet and nonSoviet Propaganda]. Abgerufen von https://ms.detector.media/trends/1411978127/ propaganda_sovetskaya_i_nesovetskaya/. Abgerufen am 13. Februar 2020.

Pochepstov Georgii (2018a). Propaganda: Osnovnye harakteristiki. [Propaganda: Key Features]. Abgerufen von https://ms.detector.media/trends/1411978127/propaganda osnovnye_kharakteristiki/. Abgerufen am 13. Februar 2020.

Pochepstov, Georgii (2018b). Metapropaganda kak dominirovanie propagandy nad ideologiej, a ne naoborot. [Meta-Propaganda as the Dominance of Propaganda over Ideology, and not vice versa]. Abgerufen von https:/hvylya.net/analytics/society/ metapropaganda-kak-dominirovanie-propagandyi-nad-ideologiey-a-ne-naoborot.html. Abgerufen am 13. Februar 2020.

Pochepstov, Georgii (2018c). Metapropaganda i propaganda: Skhodstva i razlichiya. [Meta-Propaganda and Propaganda: Similarities and Differences]. Abgerufen von https://detector.media/withoutsection/article/141356/2018-09-28-metapropaganda-i-propaganda-skhodstva-i-razlichiya/. Abgerufen am 13. Februar 2020.

Pochepstov, Georgii (2018d). Mify i narrativy propagandistskoj vojny. [Myths and Narratives of Propaganda War]. Abgerufen von https://ms.detector. media/trends/1411978127/mify_i_narrativy_propagandistskoy_voyny. Abgerufen am 13. Februar 2020.

Pochepstov, Georgii (2018e). Gibridnaya vojna v golovah i na ekrane. [Hybrid War in the Minds and on the Screen]. Abgerufen von https:/hvylya.net/analytics/politics/ gibridnaya-golova-v-golovah-i-na-ekrane.html. Abgerufen am 13. Februar 2020.

Pochepstov, Georgii (2018f). Feyki zhazhdut mesti. [Fakes crave for revenge]. Abgerufen von https://ms.detector.media/trends/1411978127/feyki_zhazhdut_mesti/.

Pochepstov, Georgii (2019). Upravlyaya kommunikaciej my odnovremenno upravlyaem mirom. [By Controlling Communication we Simultaneoulsly Control the World]. Abgerufen von https://zn.ua/SOCIUM/upravlyaya-kommunikaciey-my-odnovremennoupravlyaem-mirom-309839_.html. Abgerufen am 13. Februar 2020.

Pochepstov, Georgii (2019a). Virtualnye voiny. Feyki. [Virtual wars. Fakes]. Kharkiv: Folio.

Postman, Neil (1988). Wir amüsieren uns zu Tode: Urteilsbildung im Zeitalter der Unterhaltungsindustrie. Frankfurt am Main: Fischer Taschenbuch.

Reuters Institute (2019). Digital News Report 2019. Abgerufen von https://reuters institute.politics.ox.ac.uk/sites/default/files/inline-files/DNR_2019_FINAL.pdf. Abgerufen am 13. Februar 2020.

Shevcova, Lilia (2019). Ukraina, kak rossijskoe navazhdenie. [Ukraine as a Russian Obsession]. Abgerufen von https://www.pravda.com.ua/rus/columns/2019/04/22/7213189. Abgerufen am 13. Februar 2020. 
Shimaev, Roman; Lushnikova, Anna; Poletaeva, Polina (2019). »Rusofobskaya provokaciya«: V Moskve ocenili demonstracii gruzinskoj oppozicii v Tbilisi. [»Russophobic Provocation«: Demonstrations of the Georgian Opposition in Tbilisi were evaluated in Moscow]. Abgerufen von https://russian.rt.com/ussr/article/643180gruziya-protesty-rossiya-pravitelstvo-besporyadki.

Stopfake.org (2019). »Vostochnoe partnerstvo« - druzhba »protiv Rossii«. Pyat' fejkov o sotrudnichestve Ukrainy s ES. [»Eastern Partnetship« - Friendship »Against Russia«. Five Fakes about Cooperation between Ukraine and the EU]. Abgerufen von https://www.stopfake.org/vostochnoe-partnerstvo-druzhba-protiv-rossii-pyat-fejkov-osotrudnichestve-ukrainy-s-es/. Abgerufen am 13. Februar 2020.

Wong, Julia Carrie (2018). Instagram: From Facebook's »Best Hope« to Russian Propaganda Campaign Tool. The Guardian, 19. Dezember 2018. Abgerufen von https://www.theguardian.com/technology/2018/dec/18/instagram-facebook-russian-prop aganda-ira. Abgerufen am 13. Februar 2020.

Reporters Without Borders (2019a). 2019 World Press Freedom Index - A Cycle of Fear. Abgerufen von https://rsf.org/en/2019-world-press-freedom-index-cycle-fear. Abgerufen am 13. Februar 2020.

Reporters Without Borders (2019b). Index details. Data of press freedom ranking 2020. Abgerufen von https://rsf.org/en/ranking_table. Abgerufen am 13. Februar 2020. 
